\title{
Social Willingness to Pay, Mortality Risks and Contingent Valuation
}

\author{
Olivier Armantier ${ }^{1}$ \\ State University of New York at Stony Brook, USA \\ Nicolas Treich ${ }^{2}$ \\ Leerna-Inra, University of Toulouse, France
}

November 14, 2003

\footnotetext{
${ }^{1}$ The authors thank Antonio Bento and François Salanié together with two anonymous referees for very useful comments.

${ }^{2}$ Corresponding Author: Nicolas Treich, email: ntreich@toulouse.inra.fr. Tel: +33561 1285 14. Fax: +33 5611285 20. Address: Leerna-Inra, 21 all. de Brienne, 31042 Toulouse, France.
} 


\begin{abstract}
The Willingness-to-Pay approach is the basic justification for the use of the Contingent Valuation method to evaluate public mortality risk reduction programs. However, aggregating unweighted willingness-to-pay is a valid method only when individuals have the same marginal value of money, an unrealistic assumption in the presence of heterogeneity. We show that heterogeneity on wealth and baseline risk (respectively on risk reduction) leads to systematically overestimate (respectively underestimate) the social value of a risk reduction program. Using a recently published Contingent Valuation analysis, we find this overestimation to be quite modest though, approximately $15 \%$ in an upper bound case.
\end{abstract}

JEL classification: H43, D81, Q26

Key-Words: Willingness-to-pay, Value-of-Life, Contingent Valuation, Social Benefit, Heterogeneity. 


\section{Introduction}

Many regulatory decisions, such as the decision to control toxic substances in the environment, require to explicitly or implicitly weigh lives against money. Economists have developed several methods to explicitly place a dollar value on life-savings benefits. These methods rest upon the observation that individuals make, at the margin, some trade-offs between money and their own probability of dying. In particular, the concept of willingnessto-pay (WTP) to reduce mortality risks (see Drèze, 1962, Schelling, 1968, Jones-Lee, 1976) is certainly the most common and widely accepted criterion among economists (Viscusi, 1993; for various examples see the recent issues of the Journal of Risk and Uncertainty).

Current practices often evaluate a risk reduction public project by comparing the average individual's WTP to the per capita cost of the project. However, the WTP is expressed in dollars and not in terms of utility, so that the WTP approach does not capture individuals' changes in (expected) utility levels. As a result, simply averaging individuals' WTP is inconsistent with the maximization of an utilitarian social welfare function, except in the special case where individuals all have the same marginal utility of money. This case, however, is unrealistic and difficult to justify empirically. The WTP approach has then been intensively criticized by many scholars in Publics Economics and Environmental Economics (Boadway, 1982, Drèze and Stern, 1987, Blackorby and Donaldson, 1990, Brekke, 1997, Drèze, 1998). 
Despite the numerous criticisms, we have not found any analysis that investigates the welfare distortions created by the WTP approach. This paper is an attempt to address this question in the context of mortality risks regulation.

To understand why the WTP approach creates a welfare distortion, consider the following simple example. Two agents $i=1,2$ compose the society. Their initial wealth is $\left(w_{1}, w_{2}\right)=(1000,6000)$ and their initial survival probability $\left(p_{1}, p_{2}\right)=(0.9,0.2)$. The social project has the same cost $c=500$ for each agent. It increases their probability of survival to $\left(p_{1}+\varepsilon_{1}, p_{2}+\varepsilon_{2}\right)=(1,0.25)$. Expected utility is simply survival probability times wealth, i.e. $U_{i}=p_{i} \times w_{i}$. Thus, it is easy to show that the agents' WTP $z_{i}$ are respectively $z_{1}=-400$ and $z_{2}=700 .{ }^{1}$ Since $z_{1}+z_{2}=300>0$, the WTP criterion leads to adopt the project. However, the project is welfare reducing, since $\Delta\left(U_{1}+U_{2}\right)=1875-2100<0$, and thus should not be adopted.

This example illustrates the fact that aggregating the WTP across agents does not reflect their social valuation of the project. This is because agent 2 is not only richer, but he has also a higher probability of dying than agent 1 . Hence, relatively to agent 1 , he is willing to spend a larger amount of money to increase his chances of surviving. This heterogeneity across agents distorts the social criterion in favor of the acceptance of the project. It is a source of inefficiency since, once accepted, the project will be financed equally by individuals 1 and $2 .^{2}$

In this paper, we first explore with the help of a simple model the direc- 
tion in which the WTP approach distorts social valuation. The answer to this question critically depends on the type of heterogeneity in the population. We consider three types of heterogeneity: on wealth, baseline risk and, risk reduction. Then, based on an existing Contingent Valuation analysis, we investigate how much these different sources of heterogeneity affect the accuracy of the WTP approach as a measure of social welfare.

\section{A Simple Model}

We consider a simple model that allows us to think about the relation between the social valuation of changes in mortality risks, and heterogeneity within the population.

There are $n$ agents in the economy. Agent $i=1, . ., n$ has a wealth $w_{i}$ and a survival probability $p_{i}$. His expected utility is $p_{i} u\left(w_{i}\right)+\left(1-p_{i}\right) v\left(w_{i}\right)$, where $u($.$) and v($.$) represent respectively the utility function of wealth con-$ ditional on being alive and dead, with $u>v$. All agents have thus the same (state-dependent) utility function, an assumption that permits interpersonal comparisons of utility levels. We also assume that the utility functions are increasing, twice differentiable and concave, and that the marginal utility of money is larger when the individual is alive, i.e. $u^{\prime}>v^{\prime} \geq 0 .{ }^{3}$ A project entails a change $\varepsilon_{i}$ in the mortality risk for individual $i$ from $p_{i}$ to $p_{i}+\varepsilon_{i}$. The per capita cost of the project is $c$.

The social criterion may be described as follows. The project should be 
adopted in the economy if and only if $\Delta U=\sum_{i=1}^{n}\left[\left(p_{i}+\varepsilon_{i}\right) u\left(w_{i}-c\right)+\left(1-p_{i}-\varepsilon_{i}\right) v\left(w_{i}-c\right)-p_{i} u\left(w_{i}\right)-\left(1-p_{i}\right) v\left(w_{i}\right)\right]>0$.

We assume that the change of risk is relatively small, and that the cost of the project $c$ is negligible compared to the agents' wealth. Using a first-order Taylor development around $w_{i}$, we get that (1) is almost equivalent to

$$
\begin{aligned}
\Delta U \simeq & \sum_{i=1}^{n}\left[\left(p_{i}+\varepsilon_{i}\right)\left(u\left(w_{i}\right)-c u^{\prime}\left(w_{i}\right)\right)+\left(1-p_{i}-\varepsilon_{i}\right)\left(v\left(w_{i}\right)-c v^{\prime}\left(w_{i}\right)\right)\right. \\
& \left.\quad-p_{i} u\left(w_{i}\right)-\left(1-p_{i}\right) v\left(w_{i}\right)\right] \\
= & \sum_{i=1}^{n}\left[\varepsilon_{i}\left(u\left(w_{i}\right)-v\left(w_{i}\right)\right)-c\left(\left(p_{i}+\varepsilon_{i}\right) u^{\prime}\left(w_{i}\right)+\left(1-p_{i}-\varepsilon_{i}\right) v^{\prime}\left(w_{i}\right)\right)\right] .
\end{aligned}
$$

The social criterion for acceptance $\Delta U>0$ is thus equivalent to

$$
\bar{B}=\frac{\sum_{i} \varepsilon_{i}\left(u\left(w_{i}\right)-v\left(w_{i}\right)\right)}{\sum_{i}\left(p_{i}+\varepsilon_{i}\right) u^{\prime}\left(w_{i}\right)+\left(1-p_{i}-\varepsilon_{i}\right) v^{\prime}\left(w_{i}\right)}>c .
$$

In other words, the per capita benefit should exceed the per capita cost of the project.

Let us now turn to the WTP approach. Individual $i$ 's net WTP $z_{i}$ is defined by ${ }^{4}$

$$
\begin{aligned}
p_{i} u\left(w_{i}\right)+\left(1-p_{i}\right) v\left(w_{i}\right)= & \left(p_{i}+\varepsilon_{i}\right) u\left(w_{i}-c-z_{i}\right) \\
& +\left(1-p_{i}-\varepsilon_{i}\right) v\left(w_{i}-c-z_{i}\right) \\
\simeq & \left(p_{i}+\varepsilon_{i}\right)\left[u\left(w_{i}\right)-\left(c+z_{i}\right) u^{\prime}\left(w_{i}\right)\right] \\
& +\left(1-p_{i}-\varepsilon_{i}\right)\left[v\left(w_{i}\right)-\left(c+z_{i}\right) v^{\prime}\left(w_{i}\right)\right],
\end{aligned}
$$


so that we obtain

$$
z_{i} \simeq \frac{\varepsilon_{i}\left(u\left(w_{i}\right)-v\left(w_{i}\right)\right)}{\left(p_{i}+\varepsilon_{i}\right) u^{\prime}\left(w_{i}\right)+\left(1-p_{i}-\varepsilon_{i}\right) v^{\prime}\left(w_{i}\right)}-c .
$$

In accordance with intuition, the net $\mathrm{WTP} z_{i}$ increases with a positive change in the survival probability $\varepsilon_{i}$, decreases with the per capita cost of the project $c$, increases with the baseline risk $1-p_{i}$ and, given our assumption on $u$ and $v$, it also increases with wealth $w_{i}$ and with the curvature of $u .^{5}$ Also, note that $z_{i}$ is positive if and only if the project improves individual $i$ 's welfare. Hence, in principle, $z_{i}$ is an appropriate indicator of the impact of the project on individual $i$ 's welfare. ${ }^{6}$

The WTP criterion reduces to verify whether the net WTP is positive, or equivalently that the average WTP is larger than the per capita cost:

$$
\bar{Z}=\frac{1}{n} \sum_{i=1}^{n} \frac{\varepsilon_{i}\left(u\left(w_{i}\right)-v\left(w_{i}\right)\right)}{\left(p_{i}+\varepsilon_{i}\right) u^{\prime}\left(w_{i}\right)+\left(1-p_{i}-\varepsilon_{i}\right) v^{\prime}\left(w_{i}\right)}>c .
$$

Note that (6) is not in general equivalent to the socially efficient criterion (3). This is the case though when $p_{i}, \varepsilon_{i}$ and $w_{i}$ are independent of $i$, that is when agents are homogeneous. Less restrictively, this is the case when $\left(p_{i}+\varepsilon_{i}\right) u^{\prime}\left(w_{i}\right)+\left(1-p_{i}-\varepsilon_{i}\right) v^{\prime}\left(w_{i}\right)$ is the same for any individual $i$; that is, the WTP is an efficient criterion for the very special case in which the marginal utility of money is the same across individuals.

More generally, it is immediate that the WTP criterion will overestimate the value of the social project if and only if

$$
\begin{aligned}
& \frac{1}{n} \sum_{i=1}^{n} \frac{\varepsilon_{i}\left(u\left(w_{i}\right)-v\left(w_{i}\right)\right)}{\left(p_{i}+\varepsilon_{i}\right) u^{\prime}\left(w_{i}\right)+\left(1-p_{i}-\varepsilon_{i}\right) v^{\prime}\left(w_{i}\right)}-\frac{\sum_{i} \varepsilon_{i}\left(u\left(w_{i}\right)-v\left(w_{i}\right)\right)}{\sum_{i}\left(p_{i}+\varepsilon_{i}\right) u^{\prime}\left(w_{i}\right)+\left(1-p_{i}-\varepsilon_{i}\right) v^{\prime}\left(w_{i}\right)} \\
= & \bar{Z}-\bar{B} \geq 0 .
\end{aligned}
$$


Take for instance the parameters given in the introductory example (for which the first-order approximation is exact since utility is linear). Using (7), we get that the WTP overestimates the benefits of the project by more than a factor 2. Moreover, if the survival probability of agent 2 is lowered to $p_{2}=0.01$ instead of 0.2 , then the overestimation factor becomes 6.75 . However, consider the initial example but switch now wealth endowments between agent 1 and $2,\left(w_{1}, w_{2}\right)=(6000,1000)$. Then, one can easily show that the WTP criterion leads to underestimate the value of the project by a factor of 1.3. The question is thus: under which conditions does the WTP criterion lead to systematically overestimate or underestimate the value of the project?

Before answering this question, let us state a Lemma that will prove to be useful.

Lemma 1: Take a positive increasing (decreasing) function $g: \mathbb{R} \rightarrow \mathbb{R}$. Condition

$$
\frac{1}{n} \sum_{i=1}^{n} \frac{f\left(x_{i}\right)}{g\left(x_{i}\right)}-\frac{\frac{1}{n} \sum_{i} f\left(x_{i}\right)}{\frac{1}{n} \sum_{i} g\left(x_{i}\right)} \geq 0,
$$

holds if and only if $\mathrm{f} / \mathrm{g}$ is decreasing (increasing).

The proof is straightforward from applying the Covariance rule.

Let us now examine the separate effect of the three different sources of heterogeneity. First, consider that there is only heterogeneity on survival 
probability $p_{i}$. Then inequality $(7)$ reduces to $\frac{1}{n} \sum_{i=1}^{n} \frac{1}{\left(p_{i}+\varepsilon\right) u^{\prime}(w)+\left(1-p_{i}-\varepsilon\right) v^{\prime}(w)}-\frac{1}{\frac{1}{n} \sum_{i}\left(p_{i}+\varepsilon\right) u^{\prime}(w)+\left(1-p_{i}-\varepsilon\right) v^{\prime}(w)} \geq 0$,

which always holds under Jensen's inequality.

Consider now heterogeneity on wealth $w_{i}$ alone. Then inequality (7) reduces to the condition

$\frac{1}{n} \sum_{i=1}^{n} \frac{u\left(w_{i}\right)-v\left(w_{i}\right)}{(p+\varepsilon) u^{\prime}\left(w_{i}\right)+(1-p-\varepsilon) v^{\prime}\left(w_{i}\right)}-\frac{\sum_{i}\left(u\left(w_{i}\right)-v\left(w_{i}\right)\right)}{\sum_{i}(p+\varepsilon) u^{\prime}\left(w_{i}\right)+(1-p-\varepsilon) v^{\prime}\left(w_{i}\right)} \geq 0$,

for any $n$ and any $(p, \varepsilon)$ belonging to $[0,1]^{2}$. Applying Lemma 1 with $g(x)=$ $(p+\varepsilon) u^{\prime}(x)+(1-p-\varepsilon) v^{\prime}(x)$ and $f(x)=u(x)-v(x)$ gives the result since, under our assumptions on $u$ and $v$, the function $g$ is decreasing in $x$ and function $f / g$ is increasing in $x$.

Consider finally that the source of heterogeneity is on the change in mortality risk $\varepsilon_{i}$. Then the left-hand side of (7) is

$\frac{1}{n} \sum_{i=1}^{n} \frac{\varepsilon_{i}}{\left(p+\varepsilon_{i}\right) u^{\prime}(w)+\left(1-p-\varepsilon_{i}\right) v^{\prime}(w)}-\frac{\sum_{i} \varepsilon_{i}}{\sum_{i}\left(p+\varepsilon_{i}\right) u^{\prime}(w)+\left(1-p-\varepsilon_{i}\right) v^{\prime}(w)}$,

for any $n$ and $p \in[0,1]$. Applying Lemma 1 with $g(x)=(p+x) u^{\prime}(w)+(1-$ $p-x) v^{\prime}(w)$ and $f(x)=x$ yields that the sign of the previous expression is negative, since $g$ and $f / g$ are both increasing in $x$. Hence, this leads to the following proposition. 
Proposition 1 Assume that the project induces a "small" change in mortality risk, that its cost is uniformly distributed across the population and that agents are (state-dependent) utility maximizers, then:

- i) If there is heterogeneity across the population either on the survival probability $p_{i}$ or on wealth $w_{i}$, then the WTP criterion leads to overestimate the social value of the project of mortality risk reduction.

- ii) If there is heterogeneity on the change of the mortality risk $\varepsilon_{i}$, then the WTP criterion leads to underestimate the social value of the project of mortality risk reduction.

To understand i), consider a symmetric situation in which two identical individuals face the same survival probability $p$. In this homogenous society, the aggregate WTP for a risk reduction program is equal to the social value of the project. It is easy to verify that the social value of the project remains unchanged if the agents now face different survival probabilities of the form $p_{1}=p-\delta$ and $p_{2}=p+\delta .^{7}$ Note, however, that the WTP $z_{i}$ in equation (5) is convex in $p_{i}$. Therefore, the aggregate WTP is now larger than in the symmetric situation. In other words, the WTP criterion overestimates the social value of a project because individuals' WTP increase non-linearly with a change in initial risk-exposure. This non-linearity is related to the "dead anyway" effect (Pratt and Zeckhauser, 1996), namely that an individual with a fatal disease may want to spend his entire wealth for a small chance of cure. The effect of wealth heterogeneity may be understood intuitively by 
considering the extreme case in which the distribution of wealth is assumed to be unbounded. In this situation, it is always possible to find a small number of rich individuals willing to pay for the entire risk reduction project. In other words, there is no way to guarantee in this context that the WTP criterion will reject any socially inefficient project.

An intuition for effect ii) may be presented as follows. Suppose two identical individuals are willing to pay $\$ 1,000$ for a reduction by 1 in 1,000 of the risk of dying. Again, since there is no heterogeneity, aggregate WTP coincides with social benefit. The project has thus a $\$ 2,000$ social value. Now, suppose that instead of applying the project once to both individuals, the risk reduction project is applied twice to a single individual, so that it reduces his mortality risk by a 2 in 1,000. It is easy to see that this project has the same social value in both cases. However, once this individual has paid the $1,000 \$$ for the first unit of risk reduction, he will be poorer and less-exposed to risk. From (5), the WTP decreases when wealth or baseline risk decreases. Hence, these two effects make him less willing to pay another $\$ 1,000$ for the second unit of risk reduction. As a result, aggregate WTP will be less than $\$ 2,000$. Heterogeneity in the levels of risk reduction (0 in 1,000 for one individual and 2 in 1,000 for the other individual) thus leads to underestimate the social value of the project compared to the case of homogeneity (1 in 1,000 for both individuals). 


\section{Simulations with a Contingent Valuation Analysis}

The effects presented in Proposition 1 have been theoretical and qualitative. In the present section, we investigate from a numerical perspective the effects of heterogeneity on wealth, baseline risk and risk reduction. We thus provide some orders of magnitude for the estimation error for the social value of a risk reduction project due to the use of the WTP approach.

We calibrate our simulations on a Contingent Valuation analysis of mortality risk reduction conducted by Krupnick et al., (2002) on residents from the province of Ontario, in which the authors paid a particular attention to elicit actual WTP for risk reduction. Two groups of 630 and 300 respondents were asked their WTP to reduce by respectively 5 and 1 in 10,000 their baseline mortality risk.

Using the data provided in that study, we first calibrate a representative agent in the economy. We consider an agent with an average revenue of $\mathrm{C} \$ 60,000$ (standard deviation of $\mathrm{C} \$ 35,000)$ and an annual average baseline risk of 123/10,000 (standard deviation 123/10,000). We approximate the mean wealth for the representative respondent by the annual revenues cumulated over his lifetime. The average respondent in Krupnick et al. (2002)'s study being 54 years old, we consider an additional 25 years lifetime with a $5 \%$ annual discount factor. Given these figures, the average wealth is evaluated at $\mathrm{C} \$ 906,078$ (standard deviation of $\mathrm{C} \$ 528,548$ ). We adopt a square 
root utility function for $u$ and, to simplify, we consider that the utility $v$ conditional on death is 0 .

Krupnick et al., (2002) estimate that the average WTP for a 5/10,000 (respectively 1/10,000) risk reduction is $\mathrm{C} \$ 601$ (respectively $\mathrm{C} \$ 368$ ), while our calibrated model predicts a somewhat different WTP of C\$916 (respectively $\mathrm{C} \$ 183)$. Note, however, that the WTP in Krupnick et al. are highly non linear in risk reduction (i.e., agents are willing to contribute only 1.5 times more for a risk reduction five times larger). In contrast, our model is based on the expected utility framework, and therefore, the predicted WTP must be proportional to the risk reduction. Under these circumstances, our model cannot be expected to replicate precisely the non-linearities usually found in Contingent Valuation studies (Corso et al., 2001). ${ }^{8}$ Nevertheless, our calibrated predictions may be considered as a good approximation, within the expected utility framework, of the WTP found by Krupnick et al. (2002).

Moreover, this calibration exercise shows that the approximation "in the small" is very good. Indeed, when we calculate the WTP separately from equation (4), and from its first-order approximation in equation (5), we find a mere $\mathrm{C} \$ 0.232$ or $2.510^{-2} \%$ (respectively $\mathrm{C} \$ 0.01$ or $5.510^{-3} \%$ ) difference for a 5/10,000 (respectively $1 / 10,000$ ) risk reduction. ${ }^{9}$

To quantify the effect of heterogeneity, we generated a sample of size $10^{6}$ to precisely estimate the means $\bar{Z}$ and $\bar{B}$ in this population. Wealths $w_{i}$, and baseline risks $1-p_{i}$ were randomly and independently simulated from Lognormal distributions. ${ }^{10}$ In order to get results comparable to Krupnick et al. 
(2002)'s, we used the previously mentioned means and standard deviations to generate $w_{i}$ and $p_{i}$.

Given these underlying distributions, our main simulation result is that the mean WTP $(\bar{Z})$ is $\mathrm{C} \$ 917$, while the per capita social benefit $(\bar{B})$ is only C\$792. Hence, using data collected by Krupnick et al. (2002), our simulations suggest a $15.8 \%$ overestimation of the social benefits due to the WTP approach. ${ }^{11}$ The WTP approach would also provide an overestimated value of a statistical life equals to $\mathrm{C} \$ 917 / 0.0005=\mathrm{C} \$ 1.834$ million, while the unbiased value of a statistical life is $\$ \mathrm{C} 1.584$ million. In other words, if the per capita cost of the project lays between $\mathrm{C} \$ 792$ and $\mathrm{C} \$ 917$, the WTP criteria would lead to implement a socially inefficient project.

It is interesting to examine to which source of heterogeneity this overestimation should be mainly attributed. Graphs 1 and 2 represent the mean WTP and per capita social benefit when there is only heterogeneity on baseline risk or on wealth (the other variables are fixed to their mean values). The heterogeneity across individuals is captured by the standard deviation of the distribution from which the simulated data are drawn. These graphs clearly indicate that heterogeneity on wealth is by itself responsible for most of the difference between mean WTP and per capita social benefit. For the standard deviations found in Krupnick et al., (2002), the estimation error is about $\mathrm{C} \$ 125$ when there is wealth heterogeneity alone and a mere $\mathrm{C} \$ 1$ when 
there is baseline risk heterogeneity alone.

\section{Insert Graphs 1, 2 and 3 about here}

For the sake of completeness, we also analyzed the impact of heterogeneity on risk reduction. The simulated values $\varepsilon_{i}$ are generated from a Beta distribution with a mean of 5/10,000 and varying standard deviations. Graph 3 indicates that the effect of heterogeneity on risk reduction is rather limited. Additional simulations with larger mean risk reduction do not significantly affect the results. For instance, the distortion is in the vicinity of $1 \%$ (roughly $\mathrm{C} \$ 9$ ), for a 5/1,000 mean risk reduction and 5/1,000 standard deviation. ${ }^{12}$

Finally, we examined the effect of correlating wealth with individual's risk-exposure. Graph 4 (respectively 5) displays mean WTP and per capita social benefit as a function of the coefficient of correlation between wealth and survival probability (respectively risk reduction). ${ }^{13}$ In the absence of information on actual correlation coefficients, we were only interested in qualitative effects.

First, observe on both graphs that mean WTP is larger than per capita social benefit. This makes sense since heterogeneity on wealth dominates other sources of heterogeneity, and leads the mean WTP to overestimate per capita social benefit.

Graph 4 shows that the extent of the overestimation decreases with the coefficient of correlation between survival probability and wealth. Indeed, when the coefficient of correlation is negative, rich people face relatively 
more baseline risk than poor people. Consequently, rich people's WTP is very large compared to poor people (dead-anyway effect). Since using the WTP criterion, i.e. voting by dollars, overweighs rich people's welfare, a negative correlation leads to an important overestimation of the project. This overestimation will be consequently reduced when the coefficient of correlation becomes positive.

Insert Graphs 4 and 5 about here

Finally, Graph 5 displays an opposite effect. Indeed, a positive correlation between wealth and risk reduction implies that the project will be relatively more beneficial to rich people. As a result, rich people's WTP, and consequently mean WTP will rise with the correlation coefficient. The WTP approach, therefore, enhances the overestimation of the project when wealth and risk reduction are positively correlated.

\section{$3 \quad$ Aggregate WTP and Cost-Benefit Analysis}

We have established that maximizing the aggregate WTP in the presence of heterogeneity does not yield the same solution as maximizing the sum of expected utilities. This section briefly relates our results to the relevant literature on this topic.

Arnold Harberger (1971) presented the aggregate WTP approach as one of his three postulates for applied welfare economics: "[C]osts and benefits accruing to each member of the relevant group (e.g. a nation) should nor- 
mally be added without regard to the individual(s) to whom they accrue" (Harberger, p.785).

The main basis for this postulate is the Kaldor-Hicks compensation principle. This principle states that any project should be adopted if the gainers can potentially compensate the losers. The key word here is "potentially", meaning that the compensation needs not to be actually paid. This principle thus provides a strong theoretical basis for the use of the Contingent Valuation method.

The fundamental public economics argument underlying the compensation principle relies on the possibility for the government to implement perfect individualized lump-sum transfers. In that case, the government would choose the transfers so as to equalize the marginal value of money across the population. Aggregate WTP then indeed becomes a correct measure of social welfare. However, governments do not have the information, nor the power to implement optimal lump-sum transfers. ${ }^{14}$ This raises the question: What is the value of a risk-reducing public project under imperfect taxation policies?

Common wisdom suggests that the value of any project should be reduced under imperfect taxation. Indeed, the distortion cost from levying the taxes to finance the project should in principle be added to the costs generated by the project. Atkinson and Stern's (1974) analysis is actually consistent with this intuition. They show that public good provision is in general lower in the distortionary taxation optimum than in the lump-sum optimum (see 
Ballard and Fullerton (1992) or Myles (1995) for a survey discussions).

It is important to say that our approach is quite different from the one usually adopted in public economics. Our objective was not to compare a second-best optimum to a first-best optimum. The objective was to evaluate whether a public project financed by distortionary taxation, but evaluated by the aggregate WTP method (as if we were at the first-best optimum), will be overestimated or underestimated.

The paper has shown that, in the context of a mortality risks reduction project, there will be in general an overestimation of the project. Assuming that there is no correlation between the three sources of heterogeneity, this overestimation was found to be around $15 \%$. This is a quite modest difference compared to the various other sources of misestimation (see for instance Diamond and Hausman, 1994). Furthermore, remember that the main difference is due to wealth heterogeneity across the population, and that we have considered a uniform taxation scheme (as in Wilson, 1991; see also Brekke, 1997). Hence, the use of this extreme form of taxation presumably provides an upper bound for the misestimation, as, e.g., non-linear income taxation would clearly perform better. This observation arguably supports the idea that the unweighted aggregate WTP is a reasonable indicator in the specific Contingent Valuation analysis that we have considered. 


\section{Conclusion}

It has been known for a long time that the WTP criterion is not a reliable measure of social welfare. An extra-dollar has not the same value for everybody, and simply aggregating the WTP over the population is theoretically flawed. In practice, in the context of a mortality risk reduction project, and thus in many environmental valuation studies, this problem has not been seriously addressed.

This paper is an attempt to investigate this long-standing issue. We have considered a simple (state-dependent) expected utility model and we have assumed that all individuals face the same costs for the social project and, also, that the resulting mortality risk change is "small". Under these assumptions, we have shown theoretically that heterogeneity on wealth or survival probability (respectively, risk reduction) leads to overestimate (respectively, underestimate) the social value of a public risk reduction project. Based on a recent Contingent Valuation analysis, we have found this overestimation to be in the vicinity of $15 \%$. Compared to other potential sources of inaccuracy of the Contingent Valuation method, this source of misestimation seems rather small. However, correlations among the different forms of heterogeneity (e.g., if the program reduces rich people's mortality risks) might significantly enhance the misestimation bias, a problem that we could not examined due to our sample limitations. 


\section{Notes}

${ }^{1}$ The general formulae will be given in (4).

${ }^{2}$ Obviously, if it was possible to finance the project by making the two individuals contribute their actual WTP, efficiency would be retrieved. Yet, governments do not have, in general, the power to implement optimal (and costless) taxes. This issue is related to the optimal taxation literature, and it will be briefly discussed in Section 3.

${ }^{3}$ Viscusi and Evans (1990) provide some empirical support to this assumption.

${ }^{4}$ Again, we assume that $c+z_{i}$ is negligible compared to the agents' wealth.

${ }^{5}$ The comparative statics of survival probability, baseline risk or wealth on the WTP is well-known (see, e.g., Jones-Lee, 1976, Pratt and Zeckhauser, 1996). The comparative statics of the curvature of $u$ is examined in Eeckhoudt and Hammitt (2003) and Foncel and Treich (2003).

${ }^{6}$ We say "in principle" since in practice nothing guarantees that people reveal their actual WTP.

${ }^{7}$ This relies, of course, on the linearity of the social welfare function in the probabilities. For a related discussion, see Ulph (1982).

${ }^{8}$ To better match the WTP observed in Contingent Valuation analyses, one would need to adopt a non-expected utility approach. Although this approach clearly deserves more attention in the future, we did not adopt it here as our theoretical results in section 2 are based on the expected utility framework.

${ }^{9}$ Moreover, for a larger risk-reduction, such as 100 in a 10,000 change in risk, the first-order approximation would still be quite good: we find $\mathrm{C} \$ 18,163.3$ compared to C $\$ 18,072.3$.

${ }^{10}$ Since baseline risk is a probability, its corresponding Lognormal distribution has been truncated at 1.

${ }^{11}$ Simulations have been conducted for three other utility functions $u(x)=x^{0.25}, u(x)=$ $x^{0.75}$ and $u(x)=\log (x)$, for Normal distributions truncated at 0 , and with utility functions $v$ conditional on death equal to $k u(x)$ with $k \in[0,0.5]$. The orders of magnitude are comparable.

${ }^{12} \mathrm{Also}$, we made use of our numerical simulations to explore the effect of heterogeneity on risk aversion. We considered a power utility function $u_{i}(w)=w^{1-\gamma_{i}}$ with heterogenous relative risk aversion parameters $\gamma_{i}$. These parameters were drawn from a Beta distribution with mean 0.5 (which corresponds to a square root utility function) and standard deviations up to 0.25 . Our simulations indicate that heterogeneity on risk aversion may have a dramatic impact on the overestimation of social benefits, even larger than heterogeneity 
on wealth. In particular, the WTP overestimates the per capita social benefit by about $40 \%$ for a 0.15 standard deviation. However, such results are of little theoretical relevance since heterogeneity on preferences reintroduces the problem of interpersonal comparisons of utility levels.

${ }^{13}$ To create some correlation between the variables we consider a joint distribution belonging to the Morgenstern class of multivariate distributions with cumulative distribution function $(\mathrm{CDF}) G\left(c_{1}, c_{2}\right)=F_{1}\left(c_{1}\right) F_{2}\left(c_{2}\right)\left[1+\alpha\left(1-F_{1}\left(c_{1}\right)\right)\left(1-F_{2}\left(c_{2}\right)\right)\right]$, where $F_{1}($.$) is the$ marginal $\mathrm{CDF}$ of wealth and $F_{2}($.$) is the \mathrm{CDF}$ of the baseline risk or risk reduction. The parameter $\alpha \epsilon]-1,+1[$ determines the correlation between the two random variables. In particular, the variables are independent when $\alpha=0$.

${ }^{14}$ If the government would have perfect information, there would be actually no need for a Contingent Valuation analysis. 


\section{References}

Atkinson, Anthony B. and Nicholas H. Stern, 1974, "Pigou, taxation and public goods", Review of Economic Studies, 41, 119-28.

Ballard, Charles L. and Don Fullerton, 1992, "Distortionary taxes and the provision of public goods", Journal of Economic Perspectives, 6, 117-31.

Blackorby, Charles and David Donaldson, 1990, "A review article: The case against the use of the sum of compensating variations in cost-benefit analysis", Canadian Journal of Economics, 23, 471-94.

Boadway, Robin, 1982, "The welfare foundations of cost benefit analysis", Economic Journal, 84, 926-39.

Brekke, Kjell A., 1997, "The numéraire matters in cost-benefit analysis", Journal of Public Economics, 64, 117-23.

Corso, Phaedra S., Hammitt, James K. and John D. Graham, 2001, "Valuing mortality-risk reduction: Using visual aids to improve the validity of Contingent Valuation", Journal of Risk and Uncertainty, 23, 165-84.

Diamond, Peter A. and Jerry A. Hausman, 1994, "Contingent Valuation: Is some number better than no number?", Journal of Economic Perspectives, $8,45-64$.

Drèze, Jacques H., 1962, "L'utilité sociale d'une vie humaine", Revue Française de Recherche Opérationnelle, 22, 139-155.

Drèze, Jean, 1998, "Distribution matters in cost-benefit analysis: Comment on K.A. Brekke", Journal of Public Economics, 70, 485-88. 
Drèze, Jean and Nicholas H. Stern, 1987, "The theory of cost-benefit analysis", in Auerbach, A.J. and M. Feldstein (Eds), Handbook of Public Economics, Elsevier, North-Holland.

Eeckhoudt, Louis and James Hammitt, 2003, "Risk aversion and mortality risks", Journal of Environmental and Economics Management, forthcoming.

Foncel, Jérôme and Nicolas Treich, 2003, "Fear of ruin", mimeo, Leerna, University of Toulouse.

Harberger, Arnold C., 1971, "Three basic postulates for applied welfare economics: An interpretative essay", Journal of Economic Literature, 9, 78597.

Jones-Lee, Michael W., 1976, The Value of Life: An Economic Analysis, University of Chicago.

Krupnick, Alan, Alberini, Anna, Cropper, Maureen, Simon, Nathalie, O’Brien, Bernie, Goeree, Ronald, and Martin Heintzelman, 2002, "Age, wealth and the willingness to pay for mortality risk reductions: A Contingent Valuation survey of Ontario residents", Journal of Risk and Uncertainty, 24, $161-86$.

Myles, Gareth, 1995, Public Economics, Chapter 9: Public Goods, Cambridge, Cambridge University Press.

Pratt, John W. and Richard J. Zeckhauser, 1996, "Willingness to pay and the distribution of risk and wealth", Journal of Political Economy, 104, $747-63$. 
Schelling, Thomas C., 1968, "The life you save may be your own", in S.B. Chase, Jr., in Problems in Public Expenditure Analysis, The Brookings Institutions.

Ulph, Alistair, 1982, "The role of ex ante and ex post decisions in the valuation of life", Journal of Public Economics, 18, 266-76.

Viscusi, W. Kip and W. Evans, 1990, "Utility functions that depend on health status: Estimates and economic implications", American Economic Review, 80, 353-74.

Viscusi, W. Kip, 1993, "The value of risks to life and health", Journal of Economic Literature, 31, 1912-46.

Wilson, John D., 1991, "Optimal public good provision with limited lump-sum taxation", American Economic Review, 81, 153-66. 


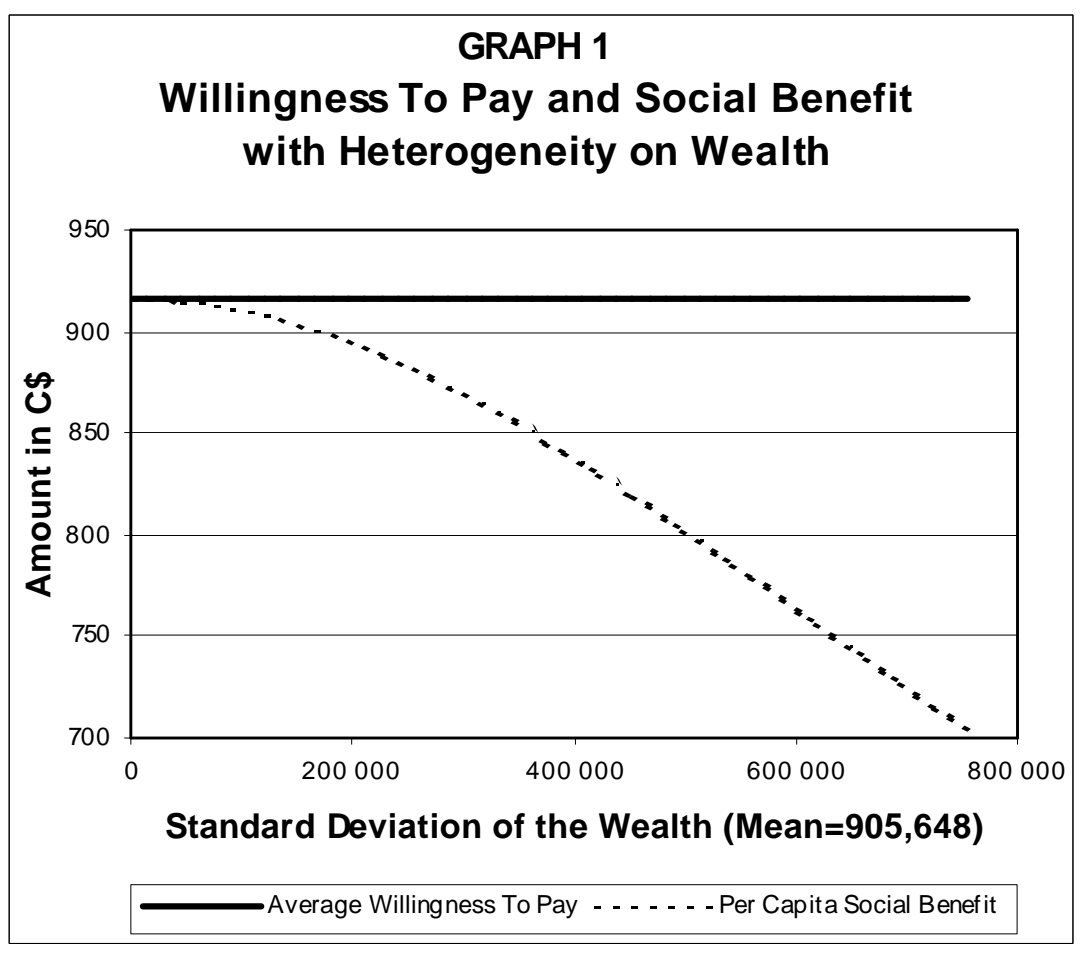




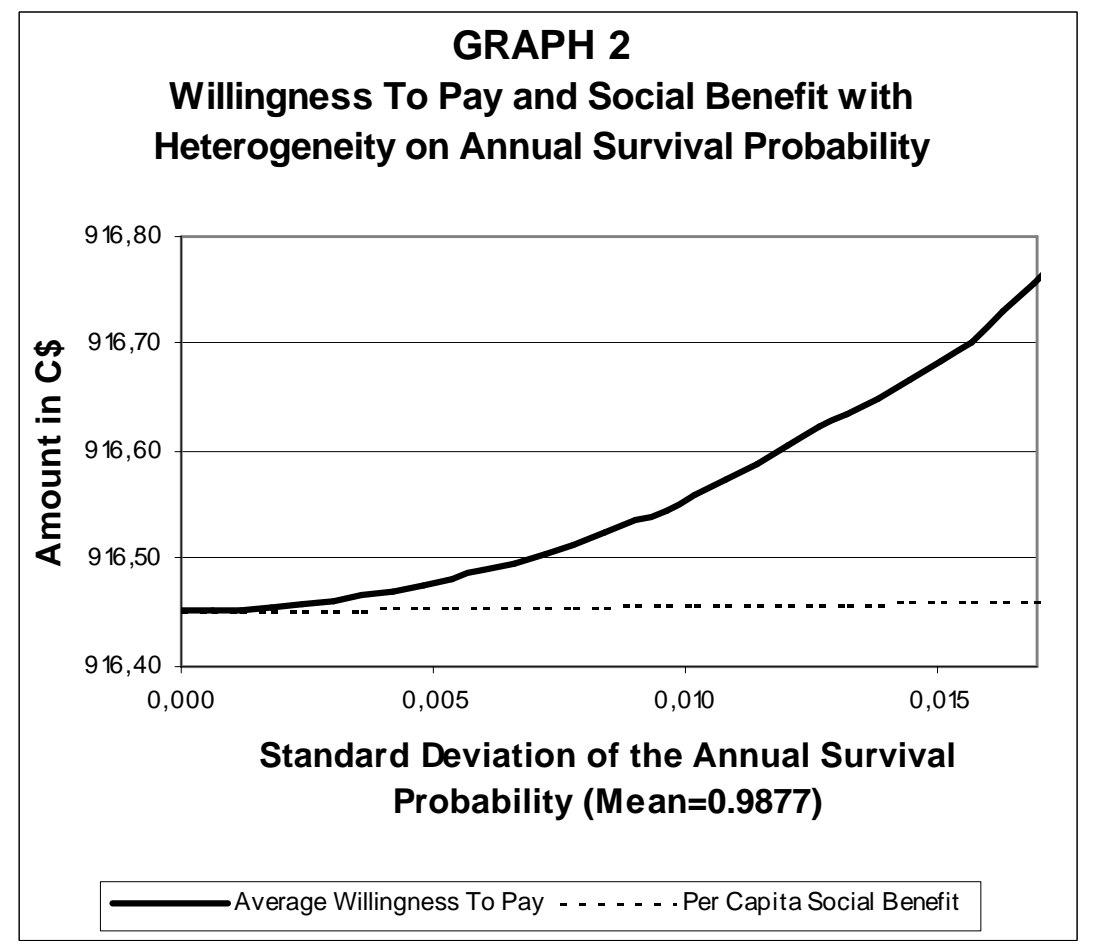




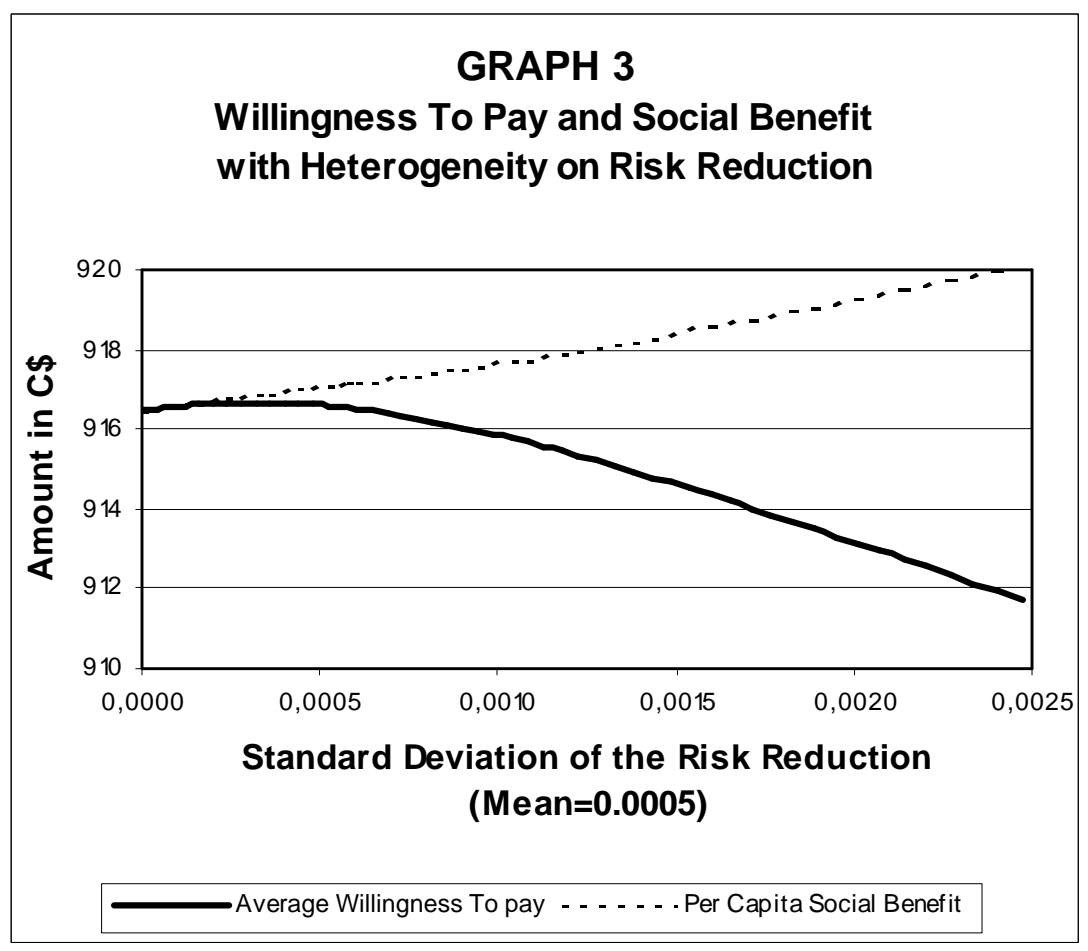




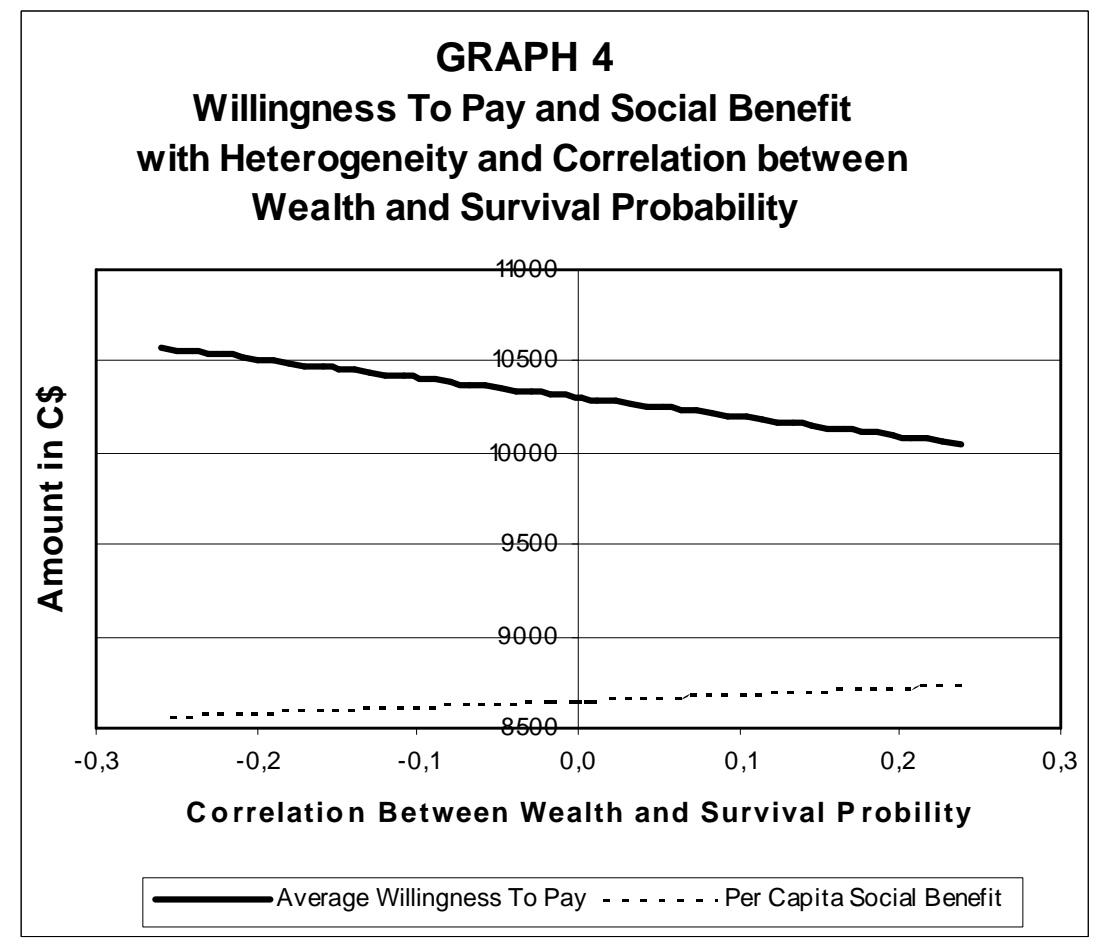




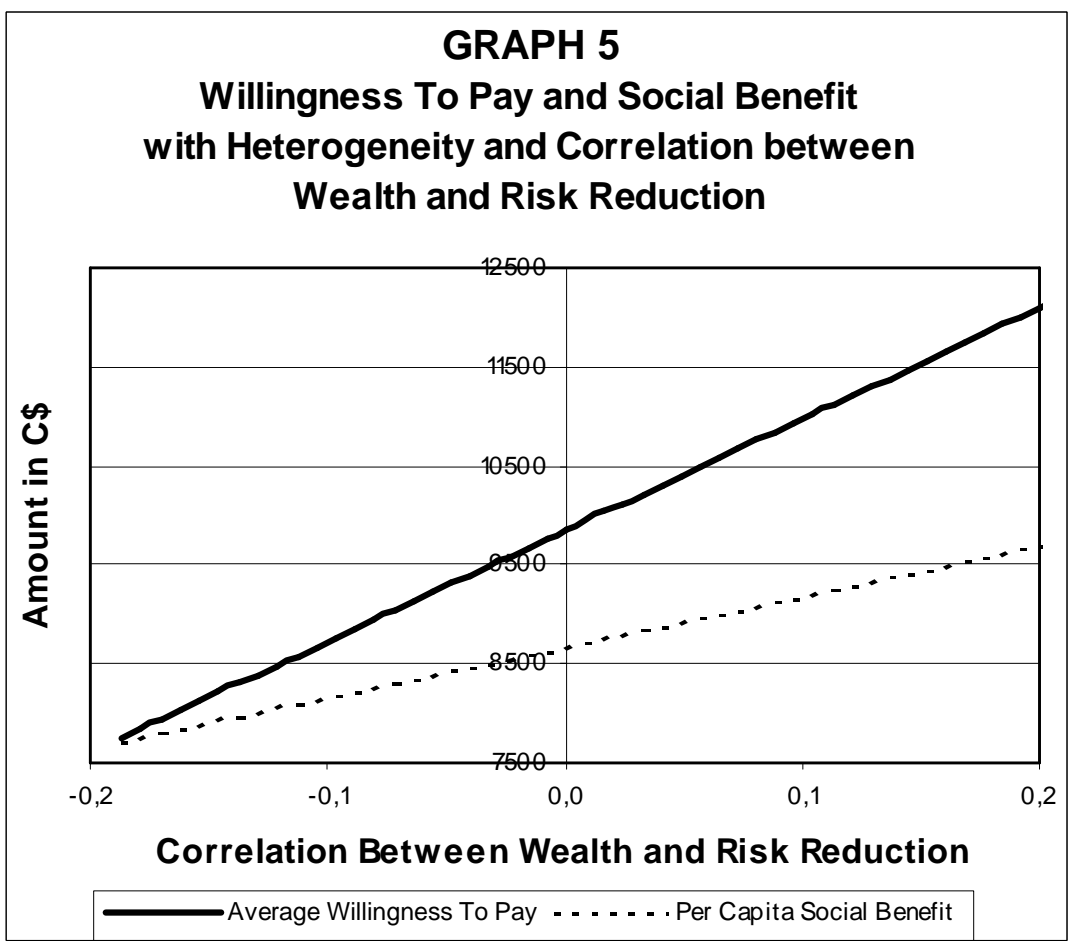

